\title{
Effectiveness and Safety of Colistin in Multi Drug Resistant Urinary Tract Infections
}

\author{
Jismy Karakkattu ${ }^{1}$, Anisha Mohan ${ }^{1}$, Emmanuel James $^{1 *}$, Anil Kumar ${ }^{2}$ \\ ${ }^{1}$ Department of Pharmacy Practice, Amrita School of Pharmacy, Amrita University, Kochi-682041, Kerala, India. \\ ${ }^{2}$ Department of Microbiology, Amrita Institute of Medical Sciences \& Research Centre, Amrita University, Kochi-682041, Kerala, India.
}

\section{ARTICLE INFO \\ Article history: \\ Received on: 24/05/2017 \\ Accepted on: 21/07/2017 \\ Available online: 30/09/2017}

\section{Key words:}

Effectiveness, colistin, MDR

bacteria, nephrotoxicity, safety, UTI.

\begin{abstract}
Colistin methanesulphonate is an inactive prodrug of which only $30 \%$ is converted to the active metabolite, colistin, in the blood and the remaining drug gets excreted unchanged in the urine. The active metabolite is eliminated through non-renal mechanisms. Hence its effectiveness in urinary tract infections (UTI) can be dubious due to the notion that no active metabolite is excreted into the bladder. But colistin has been used in clinical practice for various infections, including UTI, caused by multi drug resistant (MDR) bacteria and there is scarcity of specific reports on effectiveness of colistin in UTI. Hence the objective of our study was to evaluate the effectiveness and safety of colistin in UTI patients admitted to a tertiary care hospital. A prospective observational study was conducted on patients being treated with colistin for UTI. Safety of colistin was evaluated by assessing the causality of adverse dug reactions using Naranjo ADR probability scale and by the RIFLE criteria for severity of acute kidney injury. Clinical outcome was evaluated for each patient on completion of colistin therapy. A total of 80 patients received colistin therapy for UTI and $81 \%$ of the UTI were nosocomial. Fifty percent of UTI were relapses and urine cultures identified Klebsiella pneumoniae, Pseudomonas aeruginosa and Escherichia coli as the causative organisms, all of which were MDR. Though colistin dosages were adjusted as per creatinine clearance $36.3 \%$ of patients experienced nephrotoxicity. Clinical cure was obtained in $80 \%$ of UTI cases. Colistin is effective for treatment of UTI caused by MDR gram negative bacteria but colistin should be used judiciously due to its potential to cause nephrotoxicity.
\end{abstract}

\section{INTRODUCTION}

Polymyxins, isolated from Bacillus colistinus in the year 1949, were introduced more than 50 years ago for treatment of infections caused by gram negative bacteria (Kasiakou et al., 2005). Polymixins represent a group of five different polypeptide antibiotics (polymyxins A, B, C, D, and E) but only polymixin B and polymixin $\mathrm{E}$ (colistin) are used in clinical practice. Colistin sulphate was used orally for the treatment of infectious diarrhoea and bowl decontamination and colistimethate sodium (CMS) was

\footnotetext{
* Corresponding Author

Emmanuel James, Professor \& HOD, Department of Pharmacy Practice, Amrita School of Pharmacy, Amrita University, Kochi-682041, Kerala, India.Email: emmanuelj @ aims.amrita.edu
}

used via parenteral and inhalation routes (Falagas et al., 2005). The use of CMS later declined due to concerns of nephrotoxicity. The emergence of multi drug resistant (MDR) gram negative bacteria and the non availability of new drugs to eradicate them, forced the clinicians to start using the reserved antibiotics such as colistin. CMS is an inactive prodrug which after intravenous administration, is converted to the active metabolite, colistin, in the blood to exert its pharmacological action. But only a small proportion (30\%) of CMS is converted (Zavascki et al., 2013) to colistin in vivo. The unconverted CMS is eliminated by the kidneys via tubular secretion while the formed colistin is eliminated by non renal mechanisms (Kift et al., 2014). Hence its effectiveness in urinary tract infections (UTI) can be dubious due to the notion that no active metabolite is excreted into the bladder. But colistin has been used in clinical practice for various infections, including UTI, caused by multi drug resistant (MDR) bacteria. 
A previous report (Kasiakou et al., 2005) on use of colistin as part of combination therapy for management of various infections due to MDR gram negative bacteria in a cohort of 50 patients found that clinical cure was achieved in 6/6 (100\%) of the UTI treated. But an observational study from Israel (Paul et al., 2010) found colistin to be less effective and more toxic than beta-lactam antibiotics and associated with poor survival in patients with a variety of microbiologically documented pneumonia, UTI, surgical site infection, meningitis or bacteraemia. But specific reports on assessment of effectiveness and safety of colistin for treatment of UTI are scarce in the literature. Hence the objective of the present study was to evaluate the safety and effectiveness of colistin for UTI in patients admitted to a tertiary care hospital.

\section{MATERIALS AND METHODS}

A prospective observational cohort study was conducted in a tertiary care hospital (Amrita Institute of Medical Sciences) located in Kochi, Kerala, India after obtaining ethical clearance from the hospital Institutional Review Board. The study period was from October 2014 to May 2015. Daily census of patients receiving colistin was obtained from the pharmacy consumption report through digital hospital information system. All adult inpatients diagnosed with UTI and started treatments with colistin were included in the study. The patients should have their baseline serum creatinine levels measured prior to initiation of colistin. Patients receiving colistin for indications other than UTI and patients on dialysis were excluded from the study. Patients who were discharged against medical advice and patients/or caregivers not willing to sign informed consent were also excluded. UTI was diagnosed based on detection of pathogen in the mid stream urine sample $\left(>10^{5}\right.$ colony forming units $\left./ \mathrm{ml}\right)$ in presence of clinical symptoms (dysuria, increased frequency of urination, urgency, new incontinence and/or suprapubic pain/tenderness). Data collected included clinical signs and symptoms of UTI and elevation of lab parameters indicating possibility of infection and inflammation (temperature, WBC, and CRP). Culture and sensitivity (C/s) reports indicating UTI with causative organisms sensitive only to colistin and resistant to all other tested antibiotics resulted in colistin therapy in majority of patients as per hospital antibiotic policy. A detailed examination of the patients' medical records was conducted to gather information on patient demographics, co-morbidities, type of UTI, dose and duration of colistin and other antibiotics administered, concurrent use of potentially nephrotoxic drugs or radio contrast media and occurrence of adverse drug reactions (ADRs) and laboratory parameters including periodic serum creatinine levels and $\mathrm{C} / \mathrm{s}$ reports. Urine culture was undertaken at the hospital microbiological laboratory by trained staff. A sterile loop delivering $0.001 \mathrm{ml}$ urine was used to inoculate MacConkey and blood agar media and it was incubated overnight at $35^{\circ} \mathrm{C}$ in air. The number of colony-forming units (CFU) of each colony type was counted and categorised as $<10^{4}, 10^{4}-10^{5}$ and $>10^{5} \mathrm{CFU} / \mathrm{ml}$. A single organism at $>10^{5} \mathrm{CFU} / \mathrm{ml}$ was classified as 'significant growth' where as a single organism at $<10^{4} \mathrm{CFU} / \mathrm{ml}$ or two organisms at $<10^{5} \mathrm{CFU} / \mathrm{ml}$ was classified as 'no significant growth'. Cultures growing three or more organisms were reported as mixed growth. All significant organisms were identified and antimicrobial susceptibility testing was performed, where appropriate, using VITEK 2 system (BioMerieux, Marcy I' Etoile, France). Colistin susceptibility was determined using Clinical and Laboratory Standards Institute breakpoints for pseudomonas and acinetobacter species (Clinical and Laboratory Standards Institute 2012). Susceptibility to colistin and polymyxin B for E. coli and Klebsiella spp. were determined using breakpoints $(>2 \mathrm{mg} / \mathrm{L}$ resistant; and $\leq 2 \mathrm{mg} / \mathrm{L}$ susceptible) recommended by EUCAST (European Committee on Antimicrobial Susceptibility Testing 2014).

Multi drug resistance was defined as resistance to at least three of five classes of antibiotics which included penicillins, cephalosporins fluoroquinolones, carbapenems and aminoglycosides. The maintenance dose of colistin was adjusted as per calculated creatinine clearance based on Cockcroft-Gault equation (Cockcroft and Gault, 1976). The clinical outcome of each patient after completion of colistin therapy for UTI was described as either a favourable or unfavourable outcome (Sabuda et al., 2012). A favourable outcome was based on one or more of the following such as resolution of clinical signs and symptoms of urinary tract infections, decreasing trends of elevated temperature, WBC and CRP in addition to evidence of microbiological eradication. Unfavourable outcomes included one or more factors such as death during therapy, incomplete duration of colistin therapy and lack of microbiological eradication (culture reports, after completion of colistin therapy, showing the growth of the same organisms which were present initially in the urine sample).

Safety of colistin was assessed by monitoring the adverse drug reactions (ADRs) that may occur with colistin. Patients were continuously monitored for ADRs including nephrotoxicity, neurotoxicity and/or hypersensitivity reactions. Patients were watched for signs of neurotoxicity such as paresthesias, dizziness, and confusion. Signs such as chest tightness and breathing difficulty were also monitored. Patients were also observed for signs of hypersensitivity reactions such as facial edema, swollen tongue, rashes, and urticaria. Cusality of ADRs was evaluated using Naranjo ADR probability scale (Naranjo et al., 1981). Renal function tests, including serum urea and serum creatinine (SCR) levels were measured prior to starting colistin and continuously monitored throughout the duration of colistin therapy and also on first revisit where possible. Nephrotoxicity was defined as at least two consecutive SCR measurements with an increase of $0.5 \mathrm{mg} / \mathrm{dl}$ from baseline at least 24 hours apart after two or more days of colistin therapy (DeRyke et al., 2010). In patients with pre-existing renal dysfunction, renal failure was defined as an increase of SCR level $\geq 50 \%$ compared with baseline value. The RIFLE (Risk Injury -Failure - Loss - End stage renal disease) criteria were used to evaluate the severity of acute kidney injury (Kellum et al., 2008). One million units (MU) of CMS were considered to be 
equivalent to $80 \mathrm{mg}$ of CMS or $30 \mathrm{mg}$ of colistin base activity. Colistin consumption was calculated to defined daily dose (DDD) of $3 \mathrm{MU}$ as per the anatomic therapeutic chemical/DDD index from the WHO collaborating centre for drug statistics methodology (WHO Collaborating Centre for Drug Statistics Methodology 2012). Quantitative descriptive analysis was performed using IBM Statistical Package for Social Science (version 20) software for Windows using univariate statistics to report means and standard deviation.

\section{RESULTS AND DISCUSSION}

Out of a total of 290 patients who received colistin during the study period for various indications, colistin therapy was evaluated for UTI in 80 patients. The clinical and demographic features of the UTI patients are given in Table 1. Forty four patients $(55 \%)$ were $>60$ years of age, followed by 27 (34\%) patients in the age group 41-60 years, and 9 (11\%) patients $<40$ years of age. These patients belonged to 12 different specialties, general medicine $(28,35 \%)$ ranked first, followed by urology and neurology $(11,13.8 \%$ each). Twenty one (26\%) patients were from the surgical departments and 59 (74\%) from medical departments. Co- morbidities commonly observed in the study patients were systemic hypertension and type II diabetes mellitus. Many patients had more than one comorbid condition (Table 1).

Table 1: Clinical and demographic features of UTI patients $(n=80)$.

\begin{tabular}{ll}
\hline $\begin{array}{l}\text { Characteristics } \\
\text { Demographics }\end{array}$ & Mean \pm SD (range) or n (\%) \\
\hline Age & $59.5 \pm 15.6$ yrs ( range 19-83 yrs), \\
Males & $47(59 \%)$ \\
Comorbidities & $39(48.75)$ \\
Type II diabetes mellitus & $11(13.75)$ \\
Hypothyroidism & $06(7.5)$ \\
Asthma & $15(18.75)$ \\
Dyslipidemia & $42(52.5)$ \\
Systemic hypertension & $08(10)$ \\
Chronic liver disease & $14(17.5)$ \\
Renal failure & $80(100)$ \\
Prior antibiotic therapy &
\end{tabular}

Out of the 80 patients diagnosed with UTI, majority (65, $81 \%$ ) were having nosocomial UTI while 15 (19\%) patients had community acquired UTI. Out of the 65 patients who had nosocomial UTI, 58 were assessed to have infections acquired from other local hospitals and then transferred to our facility for further management. Majority of the UTI (40, 50\%) were due to re-infection (Figure 1). Eleven patients (13.75\%) were treated empirically while $69(86.25 \%)$ were treated as definitive therapy supported by culture reports. Colistin was used as a combination therapy with meropenem in $72(90 \%)$ patients and the remaining patients received colistin in combination with other broad spectrum antibiotics. The mean daily dose of colistin was 2 MU $\pm 1 \mathrm{MU}$ (median $=2 \mathrm{MU}$, range $=1$ to $9 \mathrm{MU}$ ) and the mean duration of therapy was $11.41 \pm 1.4$ days (median $=10$ days, range $=3$ to 14 days). The average creatinine clearance of all patients was $52 \mathrm{ml} / \mathrm{min}$ (range $=14.55-129.62 \mathrm{ml} / \mathrm{min}$ ). Colistin was administered as a $100 \mathrm{ml}$ infusion in normal saline over 30 minutes. The consumption of colistin during our study period was estimated to be 649 DDDs.

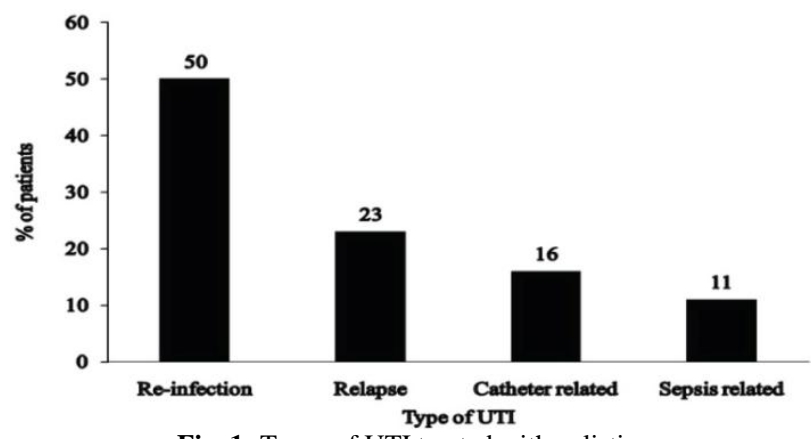

Fig. 1: Types of UTI treated with colistin.

Culture reports of urine isolates showed gram negative organisms sensitive only to colistin and resistant to all other antibiotics tested for susceptibility. The top three MDR organisms isolated were $K$. pneumoniae (53 isolates), E. coli (17 isolates), and $P$. aeruginosa (17 isolates). Twenty urine isolates identified multiple organisms consisting of a combination of $E$. coli and $K$. pneumoniae (10 isolates), Acinetobacter baumannii and E. coli (2 isoates), and $P$. aeruginosa and $K$. pneumoniae (8 isolates). All culture reports showed $>10^{5} \mathrm{CFU} / \mathrm{ml}$. The organisms isolated are represented as percentage of total isolates in Figure 2.

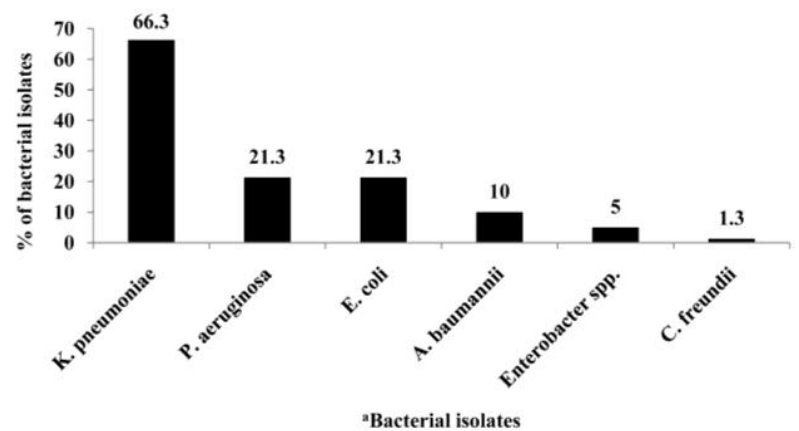

Fig. 2: Various gram negative bacteria isolated from urine specimens $(n=100)$ ${ }^{\mathrm{a}}$ Two bacteria were isolated from the urine specimens of 20 patients.

Nephrotoxicity was the only ADR observed in UTI patients treated with colistin. Nephrotoxicity as defined earlier (DeRyke et al., 2010) was manifested in 29/80 (36.3\%) patients. The mean time for onset of elevation in serum creatinine was 5.48 \pm 3.41 days (mean 5 days, median 1-18 days). As per Naranjo ADR probability scale (Naranjo et al., 1981), 18/29 (62.0\%) of the ADRs were 'Probable' and 11/29 (37.9\%) 'Possible'. As per RIFLE (Kellum et al., 2008) criteria, 13/80 (16.3\%) patients developed nephrotoxicity i.e. $7 / 80 \quad(8.8 \%)$ patients were categorized as 'risk' and 5/80 (6.3\%) categorized as 'injury' and one patient $(1.3 \%)$ as 'loss'. Fifty one $(63.7 \%)$ UTI patients treated with colistin did not experience any nephrotoxicity. The serum creatinine of 18/29 (62.0\%) patients normalized up on discontinuation of colistin. None of the patients received concomitant nephrotoxic medications or radio contrast media 
during colistin therapy. No neurotoxic adverse effects were observed in the colistin treated patients. Sixty four $(80 \%)$ patients treated with colistin had a favourable outcome while $16(20 \%)$ showed unfavourable clinical outcome. Out of the 16 patients with unfavourable outcome, 9 died during colistin therapy, 4 had incomplete therapy due to socioeconomic issues (unable to afford total cost of colistin therapy), and 3 patients did not have evidence of microbiological eradication.

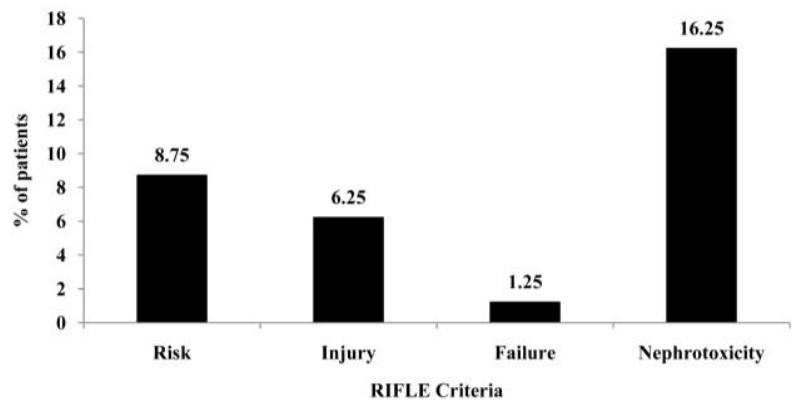

Fig. 3: UTI patients treated with colistin experiencing nephrotoxicity as per RIFLE criteria

Majority of our patients treated with colistin for UTI had a favourable outcome though they were infected with MDR pathogens which were susceptible to colistin. But nearly one third of the patients suffered renal toxicity due to the drug. Many of the patients recovered from renal dysfunction on discontinuation of colistin. None of the patients received concomitant nephrotoxic medications. No other adverse effects including neurotoxicity were observed in these patients. The incidence of renal toxicity in our study was found to be $36.3 \%$ (29/80). But reported value (Spapen $\mathrm{H}$ et al., 2011) in the literature goes up to $53.5 \%$. Colistin can cause acute tubular necrosis manifested as a rise in serum creatinine and a decrease in creatinine clearance. Nephrotoxicity is dependent on dose and duration of colistin therapy, coadministration of other nephrotoxic drugs, and patient-related factors such as age, sex, hypoalbuminemia, hyperbilirubinemia, underlying disease and severity of the patients' illness (Javan et al., 2015). The mean length of drug exposure in our study was $11.41 \pm 1.4$ days. The median dose of colistin in our study was 2 MU (range 1-9 MU). More than half of the patients (44/80, 55\%) diagnosed with UTI in our study were elderly (> 60 years). The maintenance dose of colistin was given based on calculated creatinine clearance of respective patients. A retrospective cohort study (Pogue et al., 2011) from Detroit medical centre on 126 patients treated with colistin in the same dose range as in our study but with majority in the 3-6 MU range found $43 \%$ incidence of nephrotoxicity when evaluated by RIFLE criteria. The incidence of nephrotoxicity in our study was found to be $16.75 \%$ as per RIFLE criteria. Measures such as adequate hydration, avoidance of concurrent nephrotoxic drugs and regular monitoring of renal function with appropriate dose adjustments may minimize nephrotoxicity of colistin.

Though colistin can cause neurotoxicity (Javan et al., 2015) such as facial paresthesia, ophthalmoplegia, difficulty in swallowing, ataxia, eyelid ptosis, partial deafness, visual disturbances, vertigo, confusion, hallucinations, seizures, ataxia and rarely neuromuscular blockade leading to respiratory failure, none of our patients developed any neurotoxic manifestations. Neurotoxicity was absent in other reports (Javan et al., 2015, Koomanachai et al., 2007, Levin et al., 1999, Falagas et al., 2015) of colistin therapy as well. The prevalence of neurotoxicity with colistin is considerably less as compared to its nephrotoxicity (Javan et al., 2015). Colistin was empirically administered only to 11 critically ill UTI patients, who were referred from other hospitals as part of salvage therapy. Effectiveness of colistin in UTI was assessed based on factors such as resolution of signs and symptoms of UTI, decreasing trend of elevated lab parameters towards normal levels (temperature, WBC, CRP) along with evidence of microbiological eradication of the causative bacteria as per $\mathrm{C} / \mathrm{s}$ reports showing no growth after 48 hours of discontinuation of colistin. Though only a small fraction of CMS is converted to active colistin in the blood, the unconverted CMS is excreted into the urine by tubular secretion and hydrolyzed in the aqueous system leading to high urinary concentrations of colistin (Bergen et al., 2006, Bergen et al., 2012). Hence CMS has a potential advantage in the treatment of UTI. This may be the reason for attainment of $80 \%$ cure rate of UTI in our study. This is in spite of the fact that only 10 patients in our study received a loading dose of colistin. Loading dose was suggested (Falagas et al., 2005) to attain rapidly a drug concentration equivalent to steady state plasma concentration. Physicians were nevertheless reluctant to practice this due to the fear of development of nephrotoxicity. Colistin combination therapy for MDR gram negative pneumonia was found to improve significantly the microbiological cure without improvement of clinical cure (Parchem et al., 2016). Among various drugs tested for in vitro synergy, rifampicin (Etrosillo et al., 2008) showed the maximum effect with almost $100 \%$ synergy for A. baumannii. In our study, for $69 / 80(86.25 \%)$ UTI cases, the isolated organisms were resistant to all antibiotics including meropenem. But due to the hospital policy of combination therapy, colistin was used in combination with meropenem or other broad spectrum antibiotics in our patients. Combinations of colistin with tigecyclin, minocyclin, meropenem and imipenem have been reported to be synergistic depending on the bacterial isolate tested (GiamarellosBourboulis et al., 2001, Liang et al., 2011, Souli et al., 2009, Tumbarello et al., 2012).

The cationic polypeptide ring of the colistin molecule binds to the anionic phosphate moieties in the cell membrane of the gram negative bacteria (GNB) causing displacement of calcium and magnesium ions needed for cell membrane integrity. As a result, permeability of the cell membrane increases causing leakage of cell contents and eventually cell death. The disruption of the cell membrane integrity renders the GNB susceptible to previously resistant antibiotics such as crabapenems, tetracyclines and even glycopeptides (Balaji et al., 2011). Combination therapy will not only provide a synergistic effect but also prevent development of heteroresistance to polymixins (Zusman et al., 2013). In order for the active free base to be released, the sodium 
salt must be hydrolysed at body temperature at physiological $\mathrm{pH}$ in aqueous systems (Bergen et al., 2006). It was suggested that sufficient conversion of colistin to active metabolite takes place in the bladder resulting in high urinary concentration (Bergen et al., 2006). Since high urinary concentrations of colistin are expected with parenteral administration of CMS, effectiveness of colistin monotherapy or use of colistin without a loading dose for treatment of UTI and also the influence of such a practice of monotherapy on cost effectiveness and development of resistance towards colistin needs to be explored.

\section{CONCLUSION}

Our study has confirmed the effectiveness of colistin methane sulphonate for treatment of UTI due to MDR pathogens susceptible to colistin when administered parenterally. But utilization of colistin should be done cautiously with close monitoring of serum creatinine and only for those selected patients with MDR gram negative bacterial infections due to its infamous nephrotoxic adverse effects.

\section{Financial support and sponsorship: Nil.}

Conflicts of interest: There are no conflicts of interest.

\section{REFERENCES}

Balaji V, Jeremiah SS, Baliga PR. Polymyxins: Antimicrobial susceptibility concerns and therapeutic options. Indian J Med Microbiol. 2011; 29(3): 230-42. doi: 10.4103/0255-0857.83905.

Bergen PJ, Landersdorfer CB, Zhang J Zhao M, Lee HJ, Nation RL et al. Pharmacokinetics and pharmacodynamics of 'old' polymyxins: what is new? Diagn.Microbiol. Infect, Dis. 2012; 74: 213-223.

Bergen PJ, Li J, Rayner CR, Nation RL. Colistin methanesulfonate is an inactive prodrug of colistin against Pseudomonas aeruginosa. Antimicrob Agents Chemother, 2006; 50:1953-58.

Clinical and Laboratory Standards Institute. 2012. Methods for dilution antimicrobial susceptibility tests for bacteria that grow aerobically; approved standard, 9th ed. CLSI M07-A9. Clinical and Laboratory Standards Institute, Wayne, PA.

Cockcroft, D. W., and M. H. Gault. Prediction of creatinine clearance from serum creatinine. Nephron, 1976;16: 31-41.

DeRyke CA, Crawford AJ, Uddin N, Wallace MR. Colistin Dosing and nephrotoxicity in a large community teaching hospital. Antimicrob Agents Chemother, 2010; 54: 4503-505.

Etrosillo N, Ioannidou E, Falagas ME. Colistin monotherapy vs. combination therapy: evidence from microbiological, animal and clinical studies. Clin. Microbiol. Infect, 2008; 14: 816-827

European Committee on Antimicrobial Susceptibility Testing. 2014. Breakpoint tables for interpretation of MICs and zone diameters, version 4.0. http://www.eucast.org/clinical_breakpoints/ (Accessed on $11^{\text {th }}$ September 2014).

Falagas ME, Kasiakou SK, Saravolatz LD. Colistin: the revival of polymyxins for the management of multidrug-resistant gram-negative bacterial infections. Clin Infect Dis, 2005; 40:1333-1341.

Falagas ME, Rizos M, Bliziotis IA, Rellos K, Kasiakou SK, Michalopoulos A. Toxicity after prolonged (more than four weeks) administration of intravenous colistin. BMC Infect Dis, 2005; 5:1-8.

Giamarellos-Bourboulis EJ, Xirouchaki E, Giamarellou H. Interactions of colistin and rifampin on multidrug-resistant Acinetobacter baumannii. Diagn. Microbiol. Infect. Dis, 2001; 40: 117-120.

Javan AO, Shokouhi S, Sahraei Z. A review on colistin nephrotoxicity. Eur J Clin Pharmacol, 2015; 71: 801-10.

Kasiakou S, Michalopoulos A, Soteriades E, Samonis G,
Sermaides G, Falagas ME. Combination therapy with intravenous colistin for management of infections due to multidrug-resistant gram-negative bacteria in patients without cystic fibrosis. Antimicrob Agents Chemother, 2005; 49: 3136-46.

Kellum, J. A., R. Bellomo, and C. Ronco. 2008. Definition and classification of acute kidney injury. Nephron Clin. Pract, 109:c182-c187. Kift EV, Maartens G, Bamford C. Systematic review of the evidence for rational dosing of colistin. S Afr Med J, 2014; 104:183-86.

Koomanachai P, Tiengrim S, Kiratisin P, Thamlikitkul V. Efficacy and safety of colistin (colistimethate sodium) for therapy of infections caused by multidrug-resistant Pseudomonas aeruginosa and Acinetobacter baumannii in Siriraj Hospital, Bangkok, Thailand. Int J Infect Dis 2007, 11:402-406.

Levin AS, Barone AA, Penço J, Santos MV, Marinho IS, Arruda EAG et al. Intravenous colistin as therapy for nosocomial infections caused by multidrug-resistant Pseudomonas aeruginosa and Acinetobacter baumannii. Clin Infect Dis 1999, 28:1008-1011.

Liang W, Liu XF, Huang J, Zhu DM, Li J, Zhang J. Activities of colistin- and minocycline-based combinations against extensive drug resistant Acinetobacter baumannii isolates from intensive care unit patients.. BMC Infect. Dis, 2011;11: 109

Naranjo CA, Busto U, Sellers EM, et al. A method for estimating the probability of adverse drug reactions. Clin Pharmacol Ther., 1981; 30:239-45.

Ordooei Javan AO, Shokouhi S, Sahraei Z. A review on colistin nephrotoxicity. Eur J Clin Pharmacol, 2015; 71:801-810.

Parchem NL, Bauer KA, Cook CH, Mangino JE, Jones $\mathrm{CD}$, Porter $\mathrm{K}$ etal. Colistin combination therapy improves microbiologic cure in critically ill patients with multi-drug resistant gram-negative pneumonia. , 2016; 35:1433-39.

Paul VM, Bishara J, Levcovich A, Chowers M, Goldberg E, Singer $\mathrm{P}$ et al. Effectiveness and safety of colistin: prospective comparative cohort study. J Antimicrob Chemother, 2010; 65: 1019-27.

Pogue MJ, Lee J, Marchaim D, et al. Incidence of and risk factors for colistin- associated nephrotoxicity in a large academic health system. Clinical Infectious Diseases 2011; 53:879-84.

Sabuda DM, Laupland K, Pitout J, Dalton B, Rabin H, Louie T, Conly J. Utilization of colistin for treatment of multidrugresistant Pseudomonas aeruginosa. Can J Infect Dis Med Microbiol, 2008; 19: 413-418.

Souli M, Rekatsina PD, Chryssouli Z, Galani I, Giamarellou H, Kanellakopoulou K. Does the activity of the combination of imipenem and colistin in vitro exceed the problem of resistance in metallo- $\beta$-lactamaseproducing Klebsiella pneumoniae isolates? Antimicrob. Agents Chemother, 2009; 53: 2133-2135

Spapen H, Jacobs R, Van Gorp V, Troubleyn J, Honoré PM. Renal and neurological side effects of colistin in critically ill patients. Ann Intensive Care, 2011; 1:1-7.

Tumbarello M, Viale P, Viscoli C, Trecarichi EM, Tumietto F, Marchese A et al. Predictors of mortality in bloodstream infections caused by klebsiella pneumoniae carbapenemase-producing $\mathrm{K}$. pneumoniae: importance of combination therapy. Clin Infect Dis, 2012; 55:943-50.

WHO Collaborating Centre for Drug Statistics Methodology. Guidelines for ATC classification and DDD assignment 2013. Oslo: WHO 2012.

Zavascki AP, Bulitta JB, Landersdorfer CB. Combination therapy for carbapenem- resistant gram negative bacteria. Expert Rev Anti Infect Ther, 2013; 11:1333-53

Zusman O, Avni T, Leibovici L, Adler A, Friberg $\mathrm{L}$, Stergiopoulou T et al. Systematic review and meta-analysis of in vitro synergy of polymyxins and carbapenems., 2013; 57:5104-11

How to cite this article:

Karakkattu J, Mohan A, James E, Anil Kumar. Effectiveness and Safety of Colistin In Multi Drug Resistant Urinary Tract Infections. J App Pharm Sci, 2017; 7 (09): 148-152. 